\title{
Optimal Frequency Regulation in AC Mobile Power Grids Exploiting Bilinear Matrix Inequalities
}

Javanmardi, Hamidreza; Dehghani, Maryam; Mohammadi, Mohsen; Vafamand, Navid; Dragicevic, Tomislav

Published in:

IEEE Transactions on Transportation Electrification

Link to article, DOI:

10.1109/TTE.2021.3064864

Publication date:

2021

Document Version

Peer reviewed version

Link back to DTU Orbit

Citation (APA):

Javanmardi, H., Dehghani, M., Mohammadi, M., Vafamand, N., \& Dragicevic, T. (2021). Optimal Frequency Regulation in AC Mobile Power Grids Exploiting Bilinear Matrix Inequalities. IEEE Transactions on

Transportation Electrification, 7(4), 2464 - 2473. https://doi.org/10.1109/TTE.2021.3064864

\section{General rights}

Copyright and moral rights for the publications made accessible in the public portal are retained by the authors and/or other copyright owners and it is a condition of accessing publications that users recognise and abide by the legal requirements associated with these rights.

- Users may download and print one copy of any publication from the public portal for the purpose of private study or research.

- You may not further distribute the material or use it for any profit-making activity or commercial gain

- You may freely distribute the URL identifying the publication in the public portal 


\title{
Optimal Frequency Regulation in AC Mobile Power Grids Exploiting Bilinear Matrix Inequalities
}

\author{
Hamidreza Javanmardi, Maryam Dehghani, Senior Member, IEEE, Mohsen Mohammadi, Navid \\ Vafamand, and Tomislav Dragičević, Senior Member, IEEE
}

\begin{abstract}
By day-to-day improvement in MicroGrid (MG) control, frequency regulation in the shipboard alternating current (AC) MGs has received much interest in the past decade. The shipboard MGs involve renewable energy sources (RESs) which are unable to produce reliable and constant energy. This important fact vividly demonstrates the requirement of modern frequency regulation techniques. This paper proposes a novel dynamic output feedback controller (DOFC) for load frequency control (LFC) in a sample shipboard MG. The considered AC MG involves fuel cell, gas turbine, and diesel generator as the controllable power generators; photovoltaic array, and sea wave energy converter as the renewable energy sources; and batteries and flywheels as the storage units. The existing approaches almost exploit linearization for solving the problem, which imposes restrictions on the problem and arises conservative constraints resulting in shrinking the solution space. This paper suggests a simple approach that effectively reduces such limitations through the formulation of the sufficient conditions on the controller design in the form of bilinear matrix inequalities (BMIs). These consequences in a less conservative solution compared to the previous methods. To show the merits of the developed approach, OPAL real-time (RT) simulations are executed and the results are compared to the other state-of-the-art-methods which prove the efficacy of the suggested approach. Also, the results of our proposed method (DOFC $n=2, n=3$ ) is compared to the typical $H_{\infty}$ optimization, $\mu$-synthesis, fuzzy type-1, and intelligent-PI methods. The comparisons between norms of the frequency deviation verify the efficacy of the proposed controller in this paper.
\end{abstract}

Index Terms - Shipboard AC Micro Grids; Bilinear Matrix Inequality (BMI); Dynamic Output Feedback Control (DOFC); Frequency Regulation.

\section{NOMENCLATURE}

$\begin{array}{ll}\text { BMI } & \text { Bilinear Matrix Inequalities } \\ \text { DEG } & \text { Diesel Generator }\end{array}$

H.R. Javanmardi, M. Dehghani, and N. Vafamand are with the School of Electrical and Computer Engineering, Shiraz University, Shiraz, Iran (e-mails: \{hamidjavanmardi, mdehghani, n.vafamand\}@shirazu.ac.ir).

M. Mohammadi is with the School of Mechanical Engineering, Shiraz University, Shiraz, Iran (e-mail: mohsen_mohammadi@shirazu.ac.ir).

T. Dragičević is with the Center for Electric Power and Energy, Department of Electrical Engineering, Technical University of Denmark, Lyngby, Denmark (e-mail: tomdr@elektro.dtu.dk). 
DOFC

ESS

FC

GS

LMI

MG

PV

RES

RT

S-LFC

SMG

WEC
Dynamic Output Feedback

Controller

Energy Storage System

Fuel Cell

Gas Turbine

Linear matrix inequality

Micro Grid

Photovoltaic

Renewable Energy Sources

Real-Time

Secondary Load Frequency

Control

Shipboard MG

Wave energy converter

\section{Shipboard AC MG parameter}

$\begin{array}{ll}x, A, B_{u}, B_{W}, C & \text { The state vector and the state-space } \\ & \text { matrices of the system } \\ \Delta A & \text { system uncertainty } \\ D & \text { Damping coefficient } \\ M & \text { Inertia constant } \\ \Delta f & \text { Frequency deviation } \\ T_{G T}, T_{D E G} & \text { Time constant of GT and DEG } \\ T_{F C}, T_{P V} & \text { Time constant of FC and PV } \\ T_{B E S}, T_{F E S}, T_{W E C} & \text { Time constant of BES, FES, WEC } \\ \Delta P_{G T}, \Delta P_{D E G} & \text { GT and DEG power change } \\ \Delta P_{F C}, \Delta P_{P V} & \text { FC and PV power change } \\ \Delta P_{B E S}, \Delta P_{F E S}, \Delta P_{W E C} & \text { BES, FES, WEC power change } \\ P_{L o a d} & \text { Load power }\end{array}$

\section{CONTROLLER PARAMETER}
$x_{c}, A_{c}, B_{c}, C_{c}, D_{c} \quad$ The state vector and the state-space matrices of the controller
$n_{c}$ Order of the output feedback dynamics




\section{INTRODUCTION}

$\mathrm{O}$

ver the last decades, new directions to integrate energy storage systems (ESSs) and RESs to generate power are governed due to fossil fuel shortage and high price, pollution, and environmental issues [1]-[3]. Unfortunately, RESs cannot produce constant, reliable energy, which leads to power outage or fluctuation, and if it is not compensated, the poor performance of MGs happens. This issue is even more concerning in the case of islanded MGs, such as shipboard AC MGs, than the gridconnected mode, Storage elements (e.g., flywheels, ultra-capacitors, batteries) must be allocated [4] to compensate for fluctuations in power production and result in reliable operation and high-quality of MGs.

In general, since the generated energy from the solar and wind depends on the weather situation, it is not desired to be used for the secondary frequency regulation in the islanded AC-MGs [5], [6]. The diesel engine generators (DEGs) and microturbines (MTs) afford power for the load to compensate for the energy fluctuations. Nonetheless, owing to their slow response time, they may fail to completely compensate for the unexpected fast fluctuations in renewable energy power and load. In this regard, for enhancing the overall performance of the frequency regulation mechanism, coordination with ESSs is vital to deal with the fluctuations, rapidly [7]. Matching the generated power with the load demands, characterized as load frequency control (LFC), is a crucial factor to assure the desired operation of practical power grids vulnerable to disturbances and faults.

In the secondary load frequency control (S-LFC), a robust and high-efficient control method restores the AC MG frequency to the set-point. In this branch of the AC MG control, many researches try to enhance the performance of LFC [8]. The challenges and opportunities of the secondary LFC are reviewed in [9]. In [10], a new optimal fuzzy PID control is presented for adaptive control of S-LFC in a small MG. The main idea in that work is based on fusing the fuzzy system with classical controllers. However, the authors did not consider the effects of disturbances which completely affect the model. A new modelfree sliding mode controller for the time-delayed islanded modern power grid is introduced in [11], in which, the authors use a new type of nonlinear controller to stabilize the islanded MG with electric vehicle systems. Moreover, in order to have optimal performance, a new modified population-based optimization algorithm is applied to tune the parameters of the proposed nonlinear model-free controller. In [12], a new time-varying fuzzy-PI controller is applied to the secondary frequency regulation on the AC stand-alone MG. The main disadvantage of the suggested intelligent controller is that since renewable energy sources have an inherent uncertain output, the proposed controller is not robust to the fluctuations.

Recently, great attention is devoted to approaches that formulate the S-LFC design through mathematical theories, mostly matrix inequalities [13]-[16]. In [5], [17], matrix inequalities were used to design robust $H_{\infty}$ controllers. Although the paper formulates the S-LFC problem as an LMI optimization, the approach deals with conservative methods for this transformation.

Obviously, conservatism lowers the overall performance by shrinking the design space.

A challenge in the formulation of the S-LFC problem in literature is that the main problem results in Bilinear Matrix 
Inequalities (BMI) and there are restricted approaches for dealing with BMIs. To solve BMIs, different approaches are presented including Alternate Minimization (AM) [18], [19], Inner Convex Approximation Method (ICAM) [20] Iterative linear matrix inequalities (ILMIs) [21], [22], two-step process [23], Sequential Parametric Convex Approximation (SPCA) [24], [25], Branch and Bound (BB) [26], path-following methods [27] and Method of Reduction of Variables (MRV) [28] . However, all existing approaches have some drawbacks. The BB approach is only able to compute the unknown terms in vector form, and it is not able to deal with unknown matrix variables. Also, the AM and ILMI methods divide the problem -into two separate Linear Matrix Inequalities (LMIs) and solve each separately, which surely do not converge to the unique BMI response, and there has not presented any convergence proof for them. ICAM methods are not applicable to unknown variables with special structure. The approaches SPCA and ICAM depend on a feasible initial condition and its determination is not easy. MRV formulates the problem as an structured BMI and since it is not easy to be solved, it uses heuristic algorithms for finding the optimal solution. Vividly, the approach doesn't result in convex optimization and no convergence proof can be given for the solution. Overall, all the presented approaches are highly conservative and shrink the solution space of the problem. Therefore, it still needs much effort to solve BMIs more effectively and systematically.

This paper considers the S-LFC problem of a shipboard MG (SMG) and solves the problem by a novel approach which is less conservative than the previous approaches. To regulate frequency in the system, the paper considers a nonfragile controller and tries to design the controller suitably. The nonfragile controller problem is truly in the form of a BMI problem similar to problems arising in designing less-complex controllers. Many previous approaches didn't consider its bilinear terms appropriately, even when they want to design simple controllers. This paper considers them completely and writes the BMI constraint in a simpler form through Eigen-value decomposition and change of variables. Then, in 3 different cases, based on the eigenvalues of a matrix in the BMI problem, the feasibility analysis of the BMI constraint is examined. According to these three cases, the overall optimal response is obtained.

The organization of this paper is as follows. In Section II, a brief introduction to isolated MG is presented. In Section III, the stability analysis of the closed-loop system is discussed, and sufficient conditions for designing DOFC are derived in terms of BMIs. In Section IV, a novel approach to solve BMI conditions is proposed. In Section V, simulation of DOFC for the sample shipboard AC MG is given, and comparison results are provided. By comparing the proposed method to other existing ones, the superiority of the suggested approach is highlighted. Finally, some remarking conclusions are presented in Section VI.

\section{SHIPBOARD MICROGRID MODEL}

The isolated shipboard AC MG, which is shown in Fig. 1 is considered as the system under study in this paper. A practical SMG involves LFC and automatic voltage regulator (AVR) equipment on each of its generators. Small changes in active 
(reactive) power are dependent on deviations of frequency (voltage magnitude). Thereby, due to their different sources, the LFC and AVR issues can be analyzed, independently [29]. Based on Fig. 1, the islanded AC MG includes several varying loads and energy storage systems such as flywheel energy storage (FES) and battery energy storage (BES) systems and distributed generators such as wave energy converter (WEC), photovoltaic (PV) cell, fuel cell (FC), diesel generator (DEG), and gas turbine (GT), which their power satisfy

$$
P_{\text {Load }}=\Delta P_{B E S}+\Delta P_{F E S}+\Delta P_{W E C}+\Delta P_{P V}+\Delta P_{F C}+\Delta P_{D E G}+\Delta P_{G T}
$$

Considering (1) and based on Fig. 2, the model is formulated as the following time-invariant linear space-state equation:

$$
\left\{\begin{array}{l}
\dot{x}(t)=(A+\Delta A) x(t)+B_{u} u(t)+B_{w} w(t) \\
y(t)=C x(t)
\end{array}\right.
$$

In the above model, $x \in \mathbb{R}^{n}$ is the state variable vector, $y \in \mathbb{R}^{n_{y}}$ is the measured output, $u \in \mathbb{R}^{n_{u}}$ is the input and $w \in \mathbb{R}^{n_{w}}$ is the vector of disturbances of the system, and $x(t), w(t), A, B_{u}, C$, and $B_{w}$ are given in (3) [8], and $\Delta A$ represents the system uncertainty.

$$
x(t)=\left[\begin{array}{c}
\Delta P_{W E C} \\
\Delta P_{P V} \\
\Delta P_{D E G} \\
\Delta P_{F C} \\
\Delta P_{G T} \\
\Delta P_{B E S} \\
\Delta P_{F E S} \\
\Delta f
\end{array}\right], w(t)=\left[\begin{array}{l}
\Delta P_{\text {Wave }} \\
\Delta P_{\text {Solar }} \\
\Delta P_{\text {Load }}
\end{array}\right], u(t)=\left[\begin{array}{c}
u_{D E G} \\
u_{F C} \\
u_{G T}
\end{array}\right]
$$

$$
A=\left[\begin{array}{cccccccc}
\frac{-1}{T_{W E C}} & 0 & 0 & 0 & 0 & 0 & 0 & 0 \\
0 & \frac{-1}{T_{P V}} & 0 & 0 & 0 & 0 & 0 & 0 \\
0 & 0 & \frac{-1}{T_{D E G}} & 0 & 0 & 0 & 0 & 0 \\
0 & 0 & 0 & \frac{-1}{T_{F C}} & 0 & 0 & 0 & 0 \\
0 & 0 & 0 & 0 & \frac{-1}{T_{G M}} & 0 & 0 & 0 \\
0 & 0 & 0 & 0 & 0 & \frac{-1}{T_{B E S}} & 0 & \frac{1}{T_{B E S}} \\
0 & 0 & 0 & 0 & 0 & 0 & \frac{-1}{T_{F E S}} & \frac{1}{T_{F E S}} \\
\frac{1}{M} & \frac{1}{M} & \frac{1}{M} & \frac{1}{M} & \frac{1}{M} & \frac{1}{M} & \frac{1}{M} & \frac{-}{M}
\end{array}\right],
$$




$$
B_{u}=\left[\begin{array}{c}
0 \\
0 \\
\frac{1}{T_{D E G}} \\
\frac{1}{T_{F C}} \\
\frac{1}{T_{G T}} \\
0 \\
0 \\
0
\end{array}\right], B_{w}=\left[\begin{array}{ccc}
\frac{1}{T_{W E C}} & 0 & 0 \\
0 & \frac{1}{T_{P V}} & 0 \\
0 & 0 & 0 \\
0 & 0 & 0 \\
0 & 0 & 0 \\
0 & 0 & 0 \\
0 & 0 & 0 \\
0 & 0 & -\frac{1}{M}
\end{array}\right], C=\left[\begin{array}{l}
0 \\
0 \\
0 \\
0 \\
0 \\
0 \\
0 \\
1
\end{array}\right] .
$$

The SMG frequency is controlled and regulated by the diesel generator (DEG), the gas turbine (GT), and the fuel cell (FC). The battery and the Flywheel are placed to promptly act to fast frequency variations. In addition, the power of photovoltaic (PV) cell, the power of load, and the power of the wave energy converter (WEC) are considered to be time-varying and disturbanceliked behavior, i.e., they are considered as disturbance inputs. The values of the SMG parameters are given in Table I.

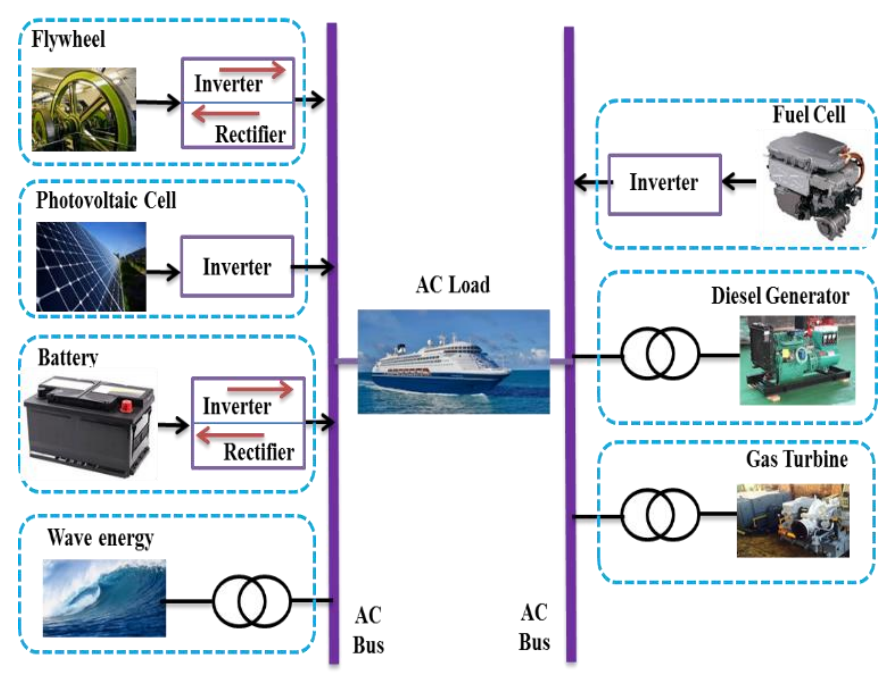

Fig. 1. Scheme of shipboard AC MG. 


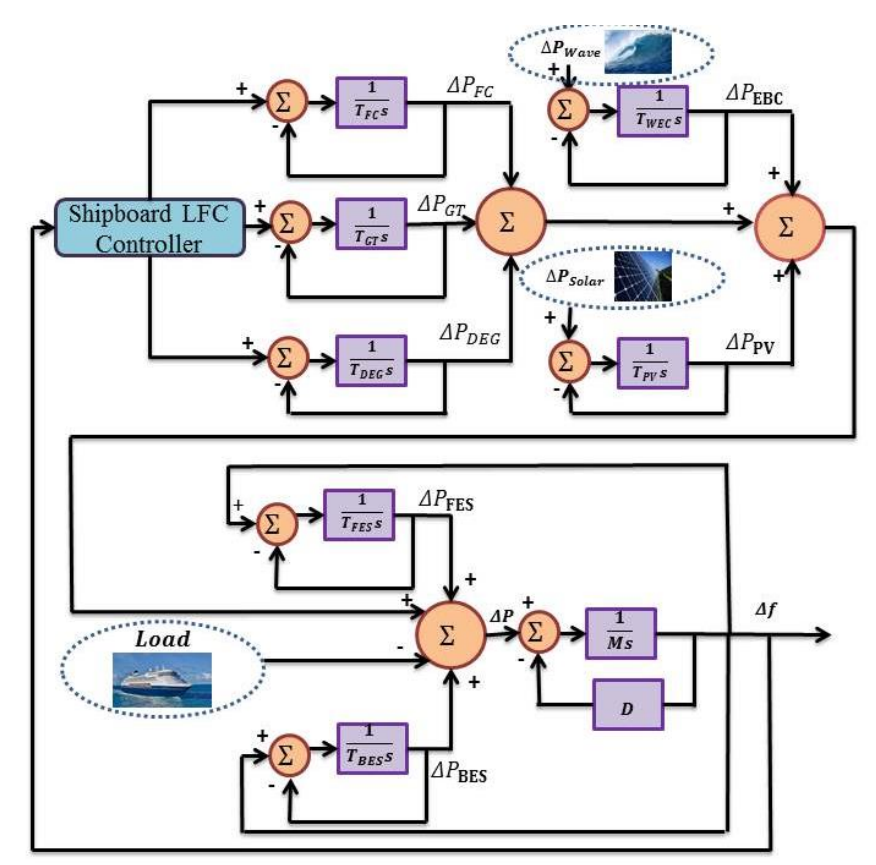

Fig. 2. The dynamic model of shipboard AC MG.

TABLE I

AC MG SYSTEM PARAMETERS VALUES [8]

\begin{tabular}{|lccc|}
\hline Param. & Value & Param. & Value \\
\hline $\boldsymbol{T}_{\boldsymbol{G T}}$ & $2 \mathrm{~s}$ & $T_{B E S}$ & $0.1 \mathrm{~s}$ \\
\hline $\boldsymbol{T}_{\boldsymbol{D E G}}$ & $2 \mathrm{~s}$ & $T_{F E S}$ & $0.1 \mathrm{~s}$ \\
\hline $\boldsymbol{T}_{\boldsymbol{F C}}$ & $4 \mathrm{~s}$ & $T_{W E C}$ & $1.5 \mathrm{~s}$ \\
\hline $\boldsymbol{T}_{\boldsymbol{P V}}$ & $1.8 \mathrm{~s}$ & $\mathrm{D}$ & $0.012(\mathrm{pu} / \mathrm{Hz})$ \\
\hline $\boldsymbol{M}$ & $0.2(p u / s)$ & & \\
\hline
\end{tabular}

\section{ROBUST NONFRAGILE DOFC DESIGN: BMI FORMULATION}

\section{A. Controller structure}

In this paper, the design of robust $H_{\infty}$ based DOFC for the system (2)-(3) to control the frequency of the SMG is proposed. Suppose the non-fragile controller has the following state-space representation:

$$
\left\{\begin{array}{l}
\dot{x}_{c}=\left(A_{c}+\Delta_{A}\right) x_{c}+\left(B_{c}+\Delta_{\mathrm{B}}\right) y \\
u=\left(C_{c}+\Delta_{C}\right) x_{c}+\left(D_{c}+\Delta_{D}\right) y
\end{array} \quad, x_{c} \in \mathbb{R}^{n_{c}}\right.
$$

The controller (4) is called non-fragile as it contains uncertainty terms $\Delta_{A}, \Delta_{B}, \Delta_{C}$, and $\Delta_{D}$. Although, the structure of the controller is completely known by the designer, controller implementation can be different from the designed one. This difference can be originated by the fact that the states are measured non-continuously and in a quantized manner, the continuous-time controller should be implemented on a digital processor with limited accuracy of the calculation. These issues affect the bound of uncertainty terms. It is considered that the controller design methodology should restrict the order of the 
controller such that it can be implemented in practice. By using the system equations (2) and controller (4), the closed-loop system equations can be represented by the following model:

$$
\left\{\begin{array}{l}
\dot{x}_{c l}=(\bar{A}+\Delta \bar{A}) x_{c l}+\bar{B} w \\
z=\bar{C} x_{c l}
\end{array} \quad, x_{c l}=\left[\begin{array}{l}
x \\
x_{c}
\end{array}\right] \in \mathbb{R}^{n+n_{c}}\right.
$$

Where

$$
\begin{gathered}
\bar{A}=\left[\begin{array}{cc}
A & 0 \\
0 & 0
\end{array}\right]+\left[\begin{array}{cc}
0 & B_{u} \\
I & 0
\end{array}\right]\left[\begin{array}{cc}
A_{c} & B_{c} \\
C_{c} & D_{c}
\end{array}\right]\left[\begin{array}{ll}
0 & I \\
C & 0
\end{array}\right]=\overline{A_{c}}+\overline{A_{l}} G_{c} \bar{A}_{r} \\
\Delta \bar{A}=\left[\begin{array}{cc}
\Delta A & 0 \\
0 & 0
\end{array}\right]+\left[\begin{array}{cc}
0 & B_{u} \\
I & 0
\end{array}\right]\left[\begin{array}{ll}
\Delta_{A} & \Delta_{B} \\
\Delta_{C} & \Delta_{D}
\end{array}\right]\left[\begin{array}{ll}
0 & I \\
C & 0
\end{array}\right] \\
\bar{B}=\left[\begin{array}{c}
B_{w} \\
0
\end{array}\right], \bar{C}=\left[\begin{array}{ll}
C & 0
\end{array}\right]
\end{gathered}
$$

where $G_{c}=\left[\begin{array}{ll}A_{c} & B_{c} \\ C_{c} & D_{c}\end{array}\right]$ contains the unknown controller dynamics, which should be found in the design procedure, while the other matrices depend on the MG model. For simplicity, assume that $\Delta A=B_{u} \Delta A_{1} C$, where $\Delta A_{1}$ is a new uncertainty system.

Therefore (6) can be written as,

$$
\Delta \bar{A}=\left[\begin{array}{cc}
0 & B_{u} \\
I & 0
\end{array}\right]\left(\left[\begin{array}{cc}
0 & 0 \\
0 & \Delta A_{1}
\end{array}\right]+\left[\begin{array}{cc}
\Delta_{A} & \Delta_{B} \\
\Delta_{C} & \Delta_{D}
\end{array}\right]\right)\left[\begin{array}{cc}
0 & I \\
C & 0
\end{array}\right]=\bar{A}_{l} \Delta_{G} \bar{A}_{r}
$$

\section{B. Controller design procedure}

In this sub-section, the robust $H_{\infty}$ control for the LFC system (2)-(3) with the non-fragile DOFC (4) is designed. The goal is to find a controller $G_{C}$ such that the closed-loop system (5) is robustly stable, and also the $\infty$-norm from disturbance input $w$ to controlled output $z$ is smaller than a specified positive number $\gamma$, (i.e. $\left\|T_{w z}\right\|_{\infty}<\gamma$ ) [30]. Fig. 3 shows the closed-loop system for the $H_{\infty}$ synthesis. In order to achieve this goal, it is necessary to satisfy the following inequality:

$$
\dot{V}+\gamma^{-1} z^{T} z-\gamma w^{T} w<0
$$

where $V$ is the Lyapunov candidate. Considering a quadratic Lyapunov function $V=x_{c l}^{T} P x_{c l}$ and substituting its time derivative along with the dynamics (5) and the uncertainty structure (7) into (8) results in:

$$
x_{c l}^{T}\left\{\left(\bar{A}+\bar{A}_{l} \Delta_{G} \bar{A}_{r}\right)^{T} P+P\left(\bar{A}+\bar{A}_{l} \Delta_{G} \bar{A}_{r}\right)+\gamma^{-1} \bar{C}^{T} \bar{C}\right\} x_{c l}+x_{c l}^{T} P \bar{B} w+w^{T} \bar{B}^{T} P x_{c l}-\gamma w^{T} w<0
$$

Assuming that the uncertainty term is bounded as $\Delta_{G}^{T} \Delta_{G}<R$ and using Lemma 2 of [31] on (9), provide:

$$
\begin{aligned}
& x_{c l}^{T}\left\{\bar{A}^{T} P+P \bar{A}+\varepsilon^{-1} P \bar{A}_{l} \bar{A}_{l}^{T} P+\varepsilon \bar{A}_{r}^{T} R \bar{A}_{r}+\gamma^{-1} \bar{C}^{T} \bar{C}\right\} x_{c l}+x_{c l}^{T} P \bar{B} w+w^{T} \bar{B}^{T} P x_{c l}-\gamma w^{T} w \\
& =\left[\begin{array}{c}
x_{c l} \\
w
\end{array}\right]^{T}\left[\begin{array}{cc}
\bar{A}^{T} P+P \bar{A}+\varepsilon^{-1} P \overline{A_{l}} \bar{A}_{l}^{T} P \\
+\varepsilon \bar{A}_{r}^{T} R \bar{A}_{r}+\gamma^{-1} \bar{C}^{T} \bar{C} \\
\bar{B}^{T} P & P \bar{B} \\
\end{array}\right]\left[\begin{array}{c}
x_{c l} \\
w
\end{array}\right]<0
\end{aligned}
$$




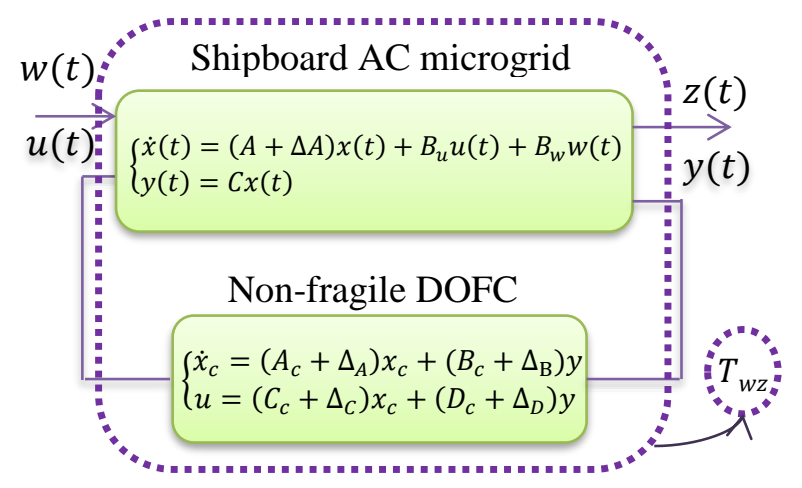

Fig. 3. $H_{\infty}$ standard configuration.

Applying Schur complement on (10), one has

$$
\left[\begin{array}{cccc}
\bar{A}^{T} P+P \bar{A}+\varepsilon \bar{A}_{r}^{T} R \bar{A}_{r} & P \bar{B} & \bar{C}^{T} & P \bar{A}_{l} \\
\bar{B}^{T} P & -\gamma I & 0 & 0 \\
\bar{C} & 0 & -\gamma I & 0 \\
\bar{A}_{l}^{T} P & 0 & 0 & \varepsilon I
\end{array}\right]<0
$$

Since the goal is to minimize the $H_{\infty}$ threshold $\gamma$, the following optimization problem with BMI constraints is obtained:

$$
\min _{G_{c}, Y, Y} \gamma
$$

Subject to (11) and $P^{T}=P>0$.

To solve the problem (12), it is needed to find a positive definite matrix $P$ such that (11) is satisfied. As it can be seen in term $\bar{A}^{T} P+P \bar{A}+\varepsilon \bar{A}_{r}^{T} R \bar{A}_{r}$, two unknown variables $P$ and $\bar{A}$ are multiplied together. Therefore, the optimization problem is in the form of a BMI. Additionally, the unknown matrices $P$ and $G_{c}$ do not appear successively and it is seen in the second term that matrix $\overline{A_{l}}$ is multiplied in the middle. All of these make challenges in solving the above BMI.

\section{PROPOSED BMI SOLVER APPROACH}

As can be seen, the DOFC design for the AC-MG system is in the form of a BMI problem. In this section, a systematic approach for solving the above BMI is presented.

Consider the BMI problem as follows:

$$
B M I(\alpha, \beta) \leq 0
$$

where

$$
B M I(\alpha, \beta)=\alpha^{T} F \beta+\beta^{T} F \alpha+H^{T} \alpha+M^{T} \beta+f
$$

In (14), $F \in \mathbb{R}^{m \times m}$ is a symmetric matrix, $H, M, f$ are appropriate constant matrices and $\alpha, \beta \in \mathbb{R}^{m \times 1}$ are unknown vectors. For solving BMI problem (13), first, this constraint is written as follows: 


$$
B M I(\alpha, \beta)=\left[\begin{array}{l}
\alpha \\
\beta
\end{array}\right]^{T} \underbrace{\left[\begin{array}{ll}
0 & F \\
F & 0
\end{array}\right]}_{\mathcal{W}} \underbrace{\left[\begin{array}{l}
\alpha \\
\beta
\end{array}\right]}_{Z}+\underbrace{\left[\begin{array}{ll}
H^{T} & H^{T}
\end{array}\right]}_{\mathcal{N}^{T}}\left[\begin{array}{l}
\alpha \\
\beta
\end{array}\right]+f=Z^{T} \mathcal{W} Z+\mathcal{N}^{T} Z+f
$$

As can be seen, eigenvalues of matrix $\mathcal{W}$ depend on the matrix $F$. So, $\mathcal{W}$ generally is an indefinite symmetric matrix. Based on Eigen-value decomposition of the symmetric matrix $\mathcal{W}$, one has:

$$
\mathcal{W}=T \Lambda T^{-1}
$$

In (16), $T \in \mathbb{R}^{m \times m}$ is the orthonormal matrix where $i$-th column of $T$ is the eigenvector $t_{i}$ of $\mathcal{W}$, and $\Lambda=\left[\begin{array}{c}{\left[a_{i}\right.} \\ 0\end{array}\left[\begin{array}{c}0 \\ -b_{i}\end{array}\right]\right.$ is the diagonal matrix which $a_{i},-b_{i}$ are the corresponding eigenvalues of $\mathcal{W}$, where $a_{i}, b_{i}$ are non-negative real numbers. By defining a new variable $\left[\begin{array}{l}X \\ Y\end{array}\right]=T^{-1} Z$, the BMI constraint (15) can be written as follows:

$$
\left[\begin{array}{ll}
X & Y
\end{array}\right]^{T} \Lambda\left[\begin{array}{l}
X \\
Y
\end{array}\right]+\mathcal{N}^{T} T\left[\begin{array}{l}
X \\
Y
\end{array}\right]+f=\sum_{i=1}^{N_{p}} a_{i} x_{i}^{2}-\sum_{j=1}^{N_{n}} b_{j} y_{j}^{2}+\sum_{i=1}^{N_{p}} c_{i} x_{i}+\sum_{j=1}^{N_{n}} d_{j} y_{j}+f<0
$$

where $X=\left[\begin{array}{lll}x_{1} & \ldots & x_{N_{p}}\end{array}\right]^{T}, Y=\left[\begin{array}{lll}y_{1} & \ldots & y_{N_{n}}\end{array}\right]^{T}, N_{p}, N_{n}$ are the number of positive and negative eigenvalues of matrix $\mathcal{W}$ respectively. To solve the problem (15), according to the eigenvalues of matrix $\mathcal{W}$, the following 3 cases are considered:

Case 1: All eigenvalues of the matrix $\mathcal{W}$ are positive (all $\left.a_{i} \neq 0, b_{j}=0\right)$ : In this case, problem (15) is a convex function which can be rewritten as follows:

$$
\sum_{i=1}^{N_{p}} a_{i} x_{i}^{2}+\sum_{i=1}^{N_{p}} c_{i} x_{i}+\sum_{j=1}^{N_{n}} d_{j} y_{j}+f<0
$$

By considering $y_{j}=\varepsilon d_{j}$, one has:

$$
\sum_{i=1}^{N_{p}} a_{i} x_{i}^{2}+\sum_{i=1}^{N_{p}} c_{i} x_{i}+\sum_{j=1}^{N_{n}} \varepsilon d_{j}^{2}+f<0
$$

The minimum value of the function $f(x)=\sum_{i=1}^{N_{p}} a_{i} x_{i}^{2}+\sum_{i=1}^{N_{p}} c_{i} x_{i}$ in (19) occurs at the point $x_{i}=\frac{c_{i}}{-2 a_{i}}$. Therefore, consider the following inequality instead of inequality (19):

$$
\varepsilon \sum_{j=1}^{N_{n}} d_{j}^{2}<\sum_{i=1}^{N_{p}} \frac{c_{i}^{2}}{4 a_{i}}-f
$$

Finally, by selecting $\varepsilon<\frac{\left(\sum_{i=1}^{N_{p}} \frac{c_{i}^{2}}{4 a_{i}}-f\right)}{\sum_{j=1}^{N_{n}} d_{j}^{2}}$, the following vector is an answer to the problem (15).

$$
Z=T\left[\begin{array}{llllll}
-\frac{c_{1}}{2 a_{1}} & \ldots & -\frac{c_{N_{p}}}{2 a_{N_{p}}} & \varepsilon d_{1} & \ldots & \varepsilon d_{N_{n}}
\end{array}\right]^{T}
$$

Case 2: All eigenvalues of the matrix $\mathcal{W}$ are negative (all $a_{i}=0, b_{j} \neq 0$ ): In this case, problem (15) is reformulated as follows: 


$$
\sum_{i=1}^{N_{p}} c_{i} x_{i}<\sum_{j=1}^{N_{n}} b_{j} y_{j}^{2}-\sum_{j=1}^{N_{n}} d_{j} y_{j}-f
$$

The minimum value of the function $f(x)=\sum_{j=1}^{N_{n}} b_{j} y_{j}^{2}-\sum_{j=1}^{N_{n}} d_{j} y_{j}$ in (22) occurs at the point $y_{j}=\frac{d_{j}}{2 b_{j}}$. Therefore, according to (22), the following equation is always valid:

$$
\sum_{i=1}^{N_{p}} c_{i} x_{i}<-\sum_{j=1}^{N_{n}} \frac{d_{j}^{2}}{4 b_{j}}-f
$$

By considering $x_{i}{ }^{\prime}=\left\{\begin{array}{cc}c_{i} x_{i} & c_{i} \neq 0 \\ 0 & c_{i}=0\end{array}\right.$ and $f^{\prime}=-\sum \frac{d_{j}^{2}}{4 b_{j}}-f$, equation (23) can be rewritten as follows:

$$
\left\|x^{\prime}\right\|_{1}<f^{\prime}
$$

where $\left\|x^{\prime}\right\|_{1}$ denotes the $H_{1}$ norm of vector $x^{\prime}$.

Finally, by solving (24), the following vector is an answer to the problem (15):

$$
Z=T\left[\begin{array}{llllll}
x_{1}^{\prime} & \ldots & x_{N_{p}}^{\prime} & \frac{d_{1}}{2 b_{1}} & \ldots & \frac{d_{N_{n}}}{2 b_{N_{n}}}
\end{array}\right]^{T}
$$

Case 3: $\mathcal{W}$ is an indefinite matrix and has both negative and positive eigenvalues (at least one of the $a_{i}$ and one of the $b_{j}$ are non-zero): In this case, it is assumed that $a_{i} \geq 0$ and $b_{j}>0$. At first, (15) is rewritten as follows:

$$
\sum_{i=1}^{N_{p}} a_{i} x_{i}^{2}+\sum_{i=1}^{N_{p}} c_{i} x_{i}<\sum_{j=1}^{N_{n}} b_{j} y_{j}^{2}-\sum_{j=1}^{N_{n}} d_{j} y_{j}-f
$$

Similar to case 1 , assuming a constant $x_{i}$ as $\bar{x}_{i}=\left\{\begin{array}{ll}\frac{c_{i}}{-2 a_{i}} & a_{i} \neq 0 \\ 0 & a_{i}=0\end{array}\right.$, inequality (26) becomes as (27):

$$
\sum_{i=1}^{N_{p}} a_{i} \bar{x}_{i}^{2}+\sum_{i=1}^{N_{p}} c_{i} \bar{x}_{i}<\sum_{j=1}^{N_{n}} b_{j} y_{j}^{2}-\sum_{j=1}^{N_{n}} d_{j} y_{j}-f
$$

So:

$$
\sum_{j=1}^{N_{n}} b_{j}\left(y_{j}-\frac{d_{j}}{2 b_{j}}\right)^{2}>f-\sum_{i=1}^{N_{p}} a_{i} \bar{x}_{i}^{2}-\sum_{i=1}^{N_{p}} c_{i} \bar{x}_{i}-\sum_{j=1}^{N_{n}} \frac{d_{j}^{2}}{4 b_{j}}
$$

By considering $y_{j}{ }^{\prime}=\sqrt{b_{j}} y_{j}-\frac{d_{j}}{2 \sqrt{b_{j}}}$ and $f^{\prime}=f-\sum_{i=1}^{N_{p}} a_{i} \bar{x}_{i}^{2}-\sum_{i=1}^{N_{p}} c_{i} \bar{x}_{i}-\sum_{j=1}^{N_{n}} \frac{d_{j}^{2}}{4 b_{j}}$, (28) can be rewritten as follows:

$$
\left\|y^{\prime}\right\|_{2}^{2}>f^{\prime}
$$

where $\left\|y^{\prime}\right\|_{2}$ denotes the $H_{2}$ norm of vector $y^{\prime}$. Finally, by solving (29), the following vector is an answer to the problem (15):

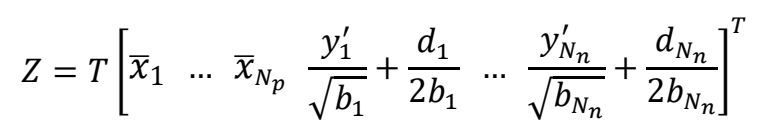


Remark 1: If at least one of the eigenvalues of the matrix $\mathcal{W}$ is negative, then there is definitely a feasible solution.

Remark 2 (advantages of the proposed approach): The developed BMI-based $H_{\infty}$ dynamic controller has the following superiorities over the state-of-the-art methods considering the same issue:

1. Comparing with LMI-based approaches [3], the proposed BMI-based controller design leads to less conservative results. This means that the closed-loop S-LFC system is more robust against the variations of the RES and load power.

2. The conventional $\mu$-synthesis and $H_{\infty}$ control methods [6] mandate a very high order of the controller. For instance, in [6], the order of the designed controller is 10 . Thereby, the computation burden of those approaches is high. On the other hand, the proposed dynamic controller not only has a smaller order than [6] but also provides better closed-loop performance.

3. In comparison with online-tuning-based methods [32], [33], and nonlinear control law with a high implementation burden, the developed approach offers an offline design procedure. Thereby, its implementation is cost-effective.

The abovementioned advantages make the proposed approach a suitable alternative for the existing LFC techniques.

The proposed algorithm for designing non-fragile DOFC for the problem (12) is summarized in Fig. 4.

\section{SIMULATION RESULTS}

In this section, the sample MG in Fig. 2 with the information given in Table I is simulated. Four scenarios are considered to show the advantages of the proposed controller over existing results and its performance against uncertainties, load power high variation and generator disconnection. By applying the proposed BMI algorithm, the control gain matrices for the DOFC are given in Table II.

\section{A. Scenario 1 (Comparative results)}

In this scenario, the non-fragile DOFC is applied to the AC MG problem and the performance of the proposed approach to minimize the effects of the external wind speed, solar radiation, and load demand variations on the frequency of the AC MG and the results are compared to the intelligent-PI [33], typical $H_{\infty}$ optimization [5], fuzzy type-1 [32] and $\mu$-synthesis [5]. Moreover, the power fluctuations $\Delta P_{\text {Load }}, \Delta P_{\text {wave }}$, and $\Delta P_{\text {Solar }}$ are shown in Fig. 5. The proposed approach approximately results in a constant frequency pattern, while the others demonstrate some fluctuations.

Fig. 6 illustrates the frequency response of the closed-loop LFC problem, by the robust $H_{\infty}$ based DOFC via BMI, typical $H_{\infty}$ optimization, $\mu$ - synthesis, fuzzy type-1, and intelligent-PI methods. As can be seen in Fig. 6, the proposed approach is more robust against power fluctuations of the RESs compared to state-of-the-art techniques. 


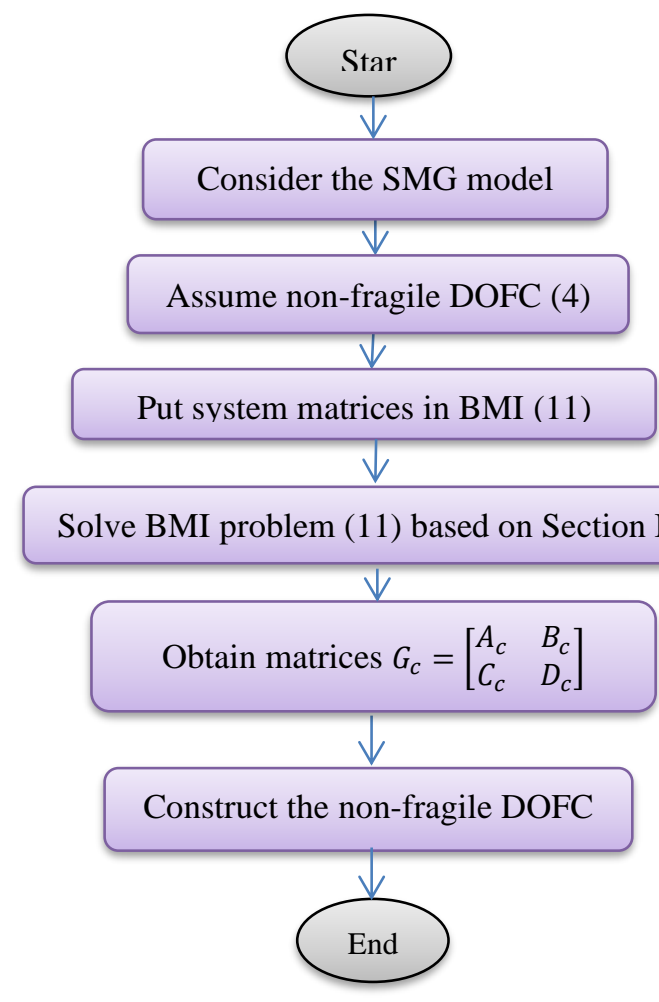

Fig. 4. The proposed algorithm for designing non-fragile DOFC.

TABLE II

Controller matrices for DOFC

\begin{tabular}{|c|c|c|c|}
\hline Ord. & 1 & 2 & 3 \\
\hline$A_{c}$ & {$[-6.450]$} & {$\left[\begin{array}{ll}-5.554 & -0.781 \\
-2.849 & -5.339\end{array}\right]$} & {$\left[\begin{array}{lll}-3.197 & -1.622 & -3.075 \\
-0.869 & -2.453 & -2.229 \\
-0.492 & -2.297 & -4.609\end{array}\right]$} \\
\hline$B_{c}$ & {$[-71.306$} & {$\left[\begin{array}{l}-43.764 \\
-59.274\end{array}\right]$} & {$\left[\begin{array}{l}-45.122 \\
-32.484 \\
-48.411\end{array}\right]$} \\
\hline$C_{c}$ & {$[-8.225]$} & {$\left[\begin{array}{lll}-5.987 & -6.989\end{array}\right]$} & {$\left[\begin{array}{llll}-4.521 & -4.012 & -6.780\end{array}\right]$} \\
\hline$D_{c}$ & -121.45 & -159.395 & -153.100 \\
\hline
\end{tabular}

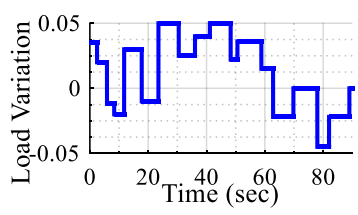

(a)

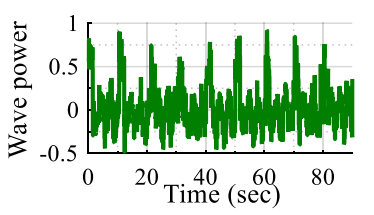

(b)

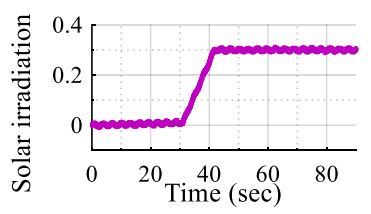

(c)

Fig. 5: Fluctuations in the load, wave converter, and PV powers [5].

The proposed approach approximately results in a constant frequency pattern, while the others demonstrate some 
fluctuations. Additionally, according to the results presented in Table III, it can be seen that the $L_{2}$ and $L_{\infty}$ norms of the proposed method are smaller compared to other controllers' results, which means the disturbances have fewer effects on the suggested approach.

The controller input signals of considered approaches are shown in Fig. 7.

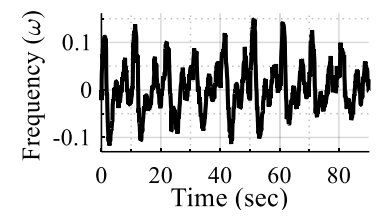

(a)

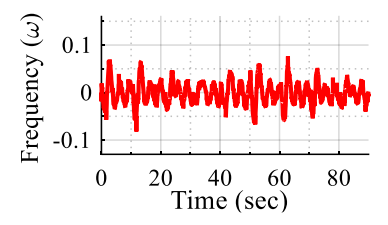

(c)

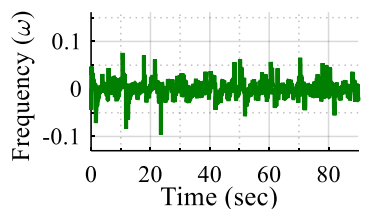

(b)

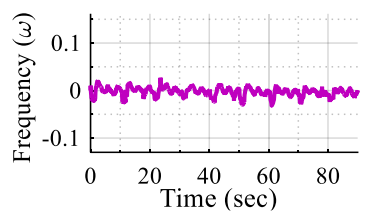

(d)

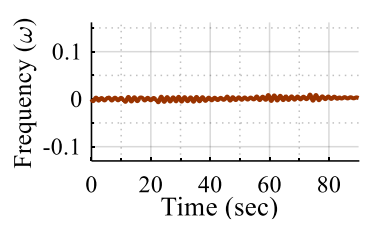

(e)

Fig. 6. Frequency response using different control approaches: (a) intelligent-PI [33], (b) fuzzy type-I [32], (c) $\mu$-approach [5],

(d) $H_{\infty}$ approach [5], (e) Non-fragile DOFC for $n_{c}=2$.

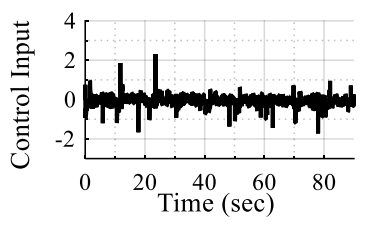

(a)

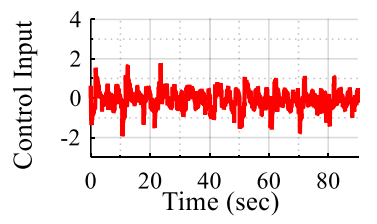

(c)

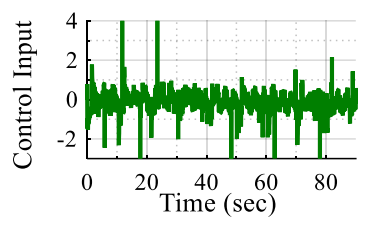

(b)

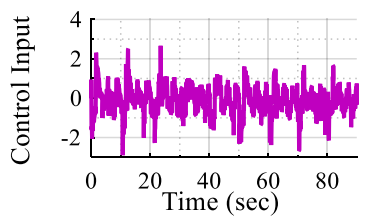

(d)

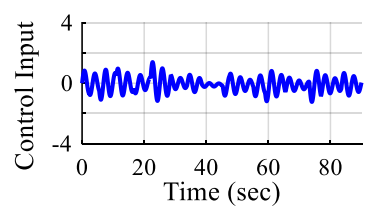

(e)

Fig. 7. Controller Signal of different control approaches: (a) intelligent-PI [33], (b) fuzzy type-I [32], (c) $\mu$-approach [5], (d) $H_{\infty}$ approach [5], (e) Non-fragile DOFC for nc=2.

Fig. 7 reveals that the proposed controller, $\mu$-synthesis [5] and typical $H_{\infty}$ optimization [5] approaches act faster when the 
SMG frequency experiences fluctuations. Thus, the closed-loop frequency response of these approaches outperforms the intelligent-PI [33], and fuzzy type-1 [32]. To the contrary, the maximum amplitude control input of the approaches DOFC, $\mu$ synthesis, and $H_{\infty}$ are almost the same as the intelligent-PI [33], and fuzzy type-1 [32].

TABLE III

NORM 2 AND $\infty$ FOR DIFFERENT APPROACHES.

\begin{tabular}{|ccc|}
\hline Approach & $\|\boldsymbol{\Delta} \boldsymbol{f}\|_{2}$ & $\|\boldsymbol{\Delta} \boldsymbol{f}\|_{\infty}$ \\
\hline DOFC $\boldsymbol{n}_{\boldsymbol{c}}=\mathbf{2}$ & 0.2781 & 0.0107 \\
\hline DOFC $\boldsymbol{n}_{\boldsymbol{c}}=\mathbf{3}$ & 0.2847 & 0.0103 \\
\hline $\boldsymbol{\mu}$-synthesis & 1.7992 & 0.0820 \\
\hline $\boldsymbol{H}_{\infty}$ & 0.7491 & 0.0345 \\
\hline PI & 4.0016 & 0.1488 \\
\hline Fuzzy type-I & 1.1650 & 0.0964 \\
\hline
\end{tabular}

\section{B. Scenario 2 (generator disconnection)}

It is assumed that each of the generators is disconnected for some interval based on the following profile:

$$
\left\{\begin{array}{l}
0 \leq t<150 \quad: \text { All generators are connected } \\
150 \leq t<250: \text { DEG } \quad \text { is disconnected } \\
250 \leq t<350: F C \quad \text { is disconnected } \\
350 \leq t<450: G T \quad \text { is disconnected }
\end{array}\right.
$$

From (31), one can see that for the time interval $0 \leq t \leq 150$ seconds, all generators are connected to the system and it operates, normally. On the other hand, in the interval $150 \leq t \leq 450$ seconds, each of the generators is disconnected for 100 seconds. The designed controller with $n_{c}=2$ is utilized in this scenario. Fig. 8(a)-(c) show the load power variation, frequency variation, and control input for this case compared to the nominal system. As can be seen in Fig. 8(b), the frequency deviation whenever DEG is disconnected is higher than the other situations and, the results of the cases in which the FC or the GT are disconnected are almost the same.

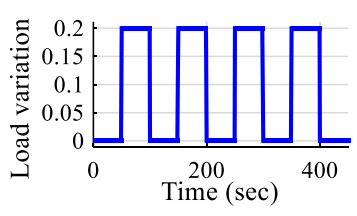

(a)

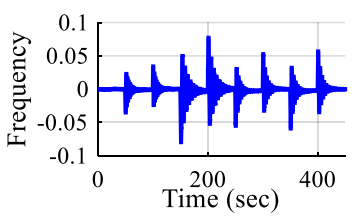

(b)

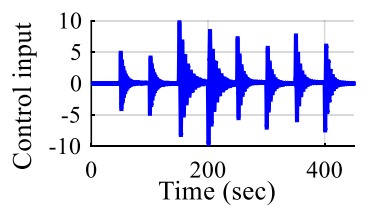

(c)

Fig. 8. The results of Scenario 2: (a) load power variation, (b) frequency deviation, and (c) control input.

\section{Scenario 3 (large load power deviation)}


Different stepwise changes in the power load are considered and the proposed controller with $n_{c}=2$ is applied to keep the frequency deviation as small as possible. The load power changes in a pulsing and ascending manner as shown in Fig. 9(a). Also, Figs. 9(b) and (c) show the frequency variation and control input for this load profile. As can be seen, by increasing the amplitude of the load power variation, the frequency deviates further. For the worst case of sudden 1 (pu) change in the load power, the frequency changes about $0.18(\mathrm{~Hz})$. This shows that the controller regulates the frequency even for the large changes in the load power.

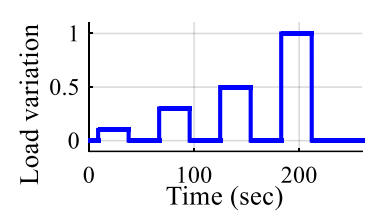

(a)

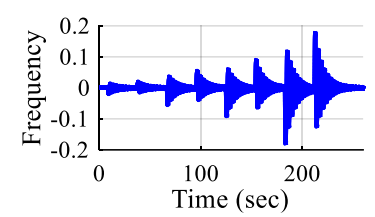

(b)

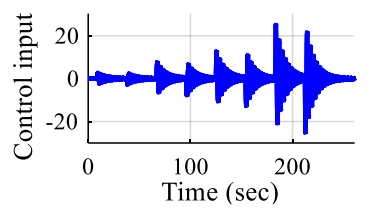

(c)

Fig. 9. The results of Scenario 3: (a) load power variation, (b) frequency deviation, and (c) control input.

\section{Scenario 4 (Parameter uncertainties)}

To evaluate the robustness of the proposed controller against the system parameter uncertainties and variations, six uncertain parameters are involved, as shown in Table IV. According to Table IV, the robustness of the proposed control scheme against the system uncertainties is evaluated by applying a variation of the parameters and the results are provided in Fig. 10(a) and (b).

TABLE IV

\begin{tabular}{|cccc|}
\multicolumn{4}{c}{ UNCERTAIN PARAMETERS OF THE MG SYSTEM } \\
\hline Parameters & Variation Range & Parameters & Variation Range \\
\hline $\boldsymbol{D}$ & $-50 \%$ & $T_{B E S}$ & $+50 \%$ \\
$\boldsymbol{M}$ & $+50 \%$ & $T_{W E C}$ & $-50 \%$ \\
$\boldsymbol{T}_{\boldsymbol{D E G}}$ & $-25 \%$ & $T_{P V}$ & $+50 \%$ \\
\hline
\end{tabular}

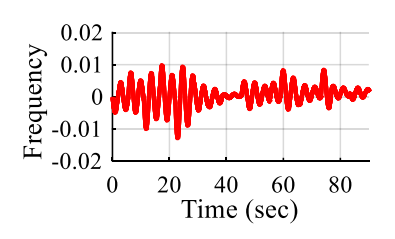

(a)

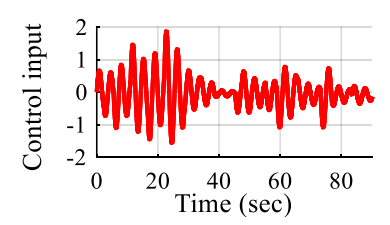

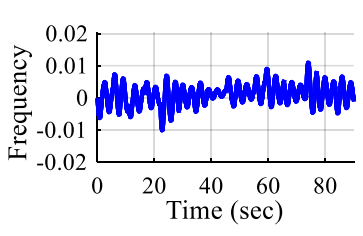

(b)

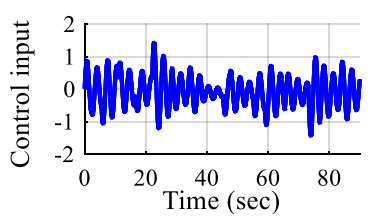


Fig. 10. The results of Scenario 4: (a) frequency deviation of uncertain system, (b) frequency deviation of nominal system, (c) control input of uncertain system, and (d) control input of nominal system.

Further, the $L_{2}$ and $L_{\infty}$ norms of the proposed method in this case are given in Table V.

TABLE V

NORM 2 AND $\infty$ FOR SCENARIO 4.

\begin{tabular}{|ccc|}
\hline Approach & $\|\boldsymbol{\Delta} \boldsymbol{f}\|_{2}$ & $\|\boldsymbol{\Delta} \boldsymbol{f}\|_{\infty}$ \\
\hline Nominal sys. & 0.2781 & 0.0107 \\
\hline Uncertain sys. & 0.2870 & 0.0127 \\
\hline
\end{tabular}

\section{CONCLUSION}

In this paper, a BMI-based dynamic output feedback controller approach was presented for load frequency regulation of an ACMG. The DOFC for the AC-MG system has been formulated as a BMI, which is robust against fluctuations in the load, wind turbine, and PV powers. A novel method was proposed to reduce the conservative conditions for solving the BMI problem. The main feature of the proposed method was that it could decompose the original BMI to a BMI problem and an LMI problem, and then it reduces the dimension and complexity of the iterative algorithm. The results illustrated that the proposed method improves the performance of the frequency response against existing controllers such as $H_{\infty}, \mu$, fuzzy type-1, and intelligent-PI methods, based on the norms 2 and $\infty$ indices. These performance improvements increase the lifetime of the batteries and flywheels by reducing their charging/discharging actions. Also, the effects of uncertainty and generator disconnection on the closed-loop system are evaluated. It means that the controller is easily implemented, and the proposed approach can be used in practice. In future work, a data driven, model free method for load frequency control (LFC) is suggested. Additionally, some issues such as time-delay, faults and short circuit should in involved to make the proposed approach more reliable.

\section{REFERENCES}

[1] J. Hou, J. Sun, and H. Hofmann, "Control development and performance evaluation for battery/flywheel hybrid energy storage solutions to mitigate load fluctuations in all-electric ship propulsion systems," Appl. Energy, vol. 212, pp. 919-930, Feb. 2018, doi: 10.1016/j.apenergy.2017.12.098.

[2] M.-H. Khooban et al., "Robust Frequency Regulation in Mobile Microgrids: HIL Implementation," IEEE Syst. J., vol. 13, no. 4, pp. 4281-4291, Dec. 2019, doi: 10.1109/JSYST.2019.2911210.

[3] S. Khosravi, M. T. Hamidi Beheshti, and H. Rastegar, "Robust Control of Islanded Microgrid Frequency Using Fractional-Order PID," Iran. J. Sci. Technol. Trans. Electr. Eng., vol. 44, no. 3, pp. 1207-1220, Sep. 2020, doi: 10.1007/s40998-019-00303-6.

[4] N. Vafamand, M. H. Khooban, T. Dragicevic, J. Boudjadar, and M. H. Asemani, "Time-Delayed Stabilizing Secondary Load Frequency Control of Shipboard Microgrids," IEEE Syst. J., vol. 13, no. 3, pp. 3233-3241, Sep. 2019, doi: 10.1109/JSYST.2019.2892528.

[5] H. Bevrani, M. R. Feizi, and S. Ataee, "Robust Frequency Control in an Islanded Microgrid: $H_{\infty}$ and $\mu$-Synthesis Approaches," IEEE Trans. Smart Grid, vol. 7, no. 2, pp. 706-717, Mar. 2016, doi: 10.1109/TSG.2015.2446984. 
[6] P. Shah and B. Mehta, "Microgrid Optimal Scheduling with Renewable Energy Sources Considering Islanding Constraints," Iran. J. Sci. Technol. Trans. Electr. Eng., vol. 44, no. 2, pp. 805-819, Jun. 2020, doi: 10.1007/s40998-019-00254-y.

[7] F. D. Kanellos, "Optimal Power Management With GHG Emissions Limitation in All-Electric Ship Power Systems Comprising Energy Storage Systems," IEEE Trans. Power Syst., vol. 29, no. 1, pp. 330-339, Jan. 2014, doi: 10.1109/TPWRS.2013.2280064.

[8] K. Torabi-Farsani, M. H. Asemani, F. Badfar, N. Vafamand, and M. H. Khooban, "Robust Mixed $\mu$-Synthesis Frequency Regulation in AC Mobile Power Grids," IEEE Trans. Transp. Electrification, vol. 5, no. 4, pp. 1182-1189, Dec. 2019, doi: 10.1109/TTE.2019.2960637.

[9] H. Alhelou, M.-E. Hamedani-Golshan, R. Zamani, E. Heydarian-Forushani, and P. Siano, "Challenges and Opportunities of Load Frequency Control in Conventional, Modern and Future Smart Power Systems: A Comprehensive Review,” Energies, vol. 11, no. 10, p. 2497, Sep. 2018, doi: $10.3390 /$ en11102497.

[10] D. Tripathy, A. K. Barik, N. B. D. Choudhury, and B. K. Sahu, "Performance Comparison of SMO-Based Fuzzy PID Controller for Load Frequency Control," in Soft Computing for Problem Solving, vol. 817, J. C. Bansal, K. N. Das, A. Nagar, K. Deep, and A. K. Ojha, Eds. Singapore: Springer Singapore, 2019, pp. 879-892.

[11] H. Li, X. Wang, and J. Xiao, “Adaptive Event-Triggered Load Frequency Control for Interconnected Microgrids by Observer-Based Sliding Mode Control,” IEEE Access, vol. 7, pp. 68271-68280, 2019.

[12] M. H. Khooban, T. Niknam, and M. Sha-Sadeghi, "Speed control of electrical vehicles: a time-varying proportional-integral controller-based type-2 fuzzy logic,” IET Sci. Meas. Technol., vol. 10, no. 3, pp. 185-192, May 2016, doi: 10.1049/iet-smt.2015.0033.

[13] M. Dehghani and S. K. Y. Nikravesh, “Robust Tuning of PSS Parameters Using the Linear Matrix Inequalities Approach,” in 2007 IEEE Lausanne Power Tech, Jul. 2007, pp. 322-326, doi: 10.1109/PCT.2007.4538337.

[14] S. Wen, X. Yu, Z. Zeng, and J. Wang, "Event-Triggering Load Frequency Control for Multiarea Power Systems With Communication Delays," IEEE Trans. Ind. Electron., vol. 63, no. 2, pp. 1308-1317, Feb. 2016, doi: 10.1109/TIE.2015.2399394.

[15] L. Jiang, W. Yao, Q. H. Wu, J. Y. Wen, and S. J. Cheng, "Delay-Dependent Stability for Load Frequency Control With Constant and Time-Varying Delays," IEEE Trans. Power Syst., vol. 27, no. 2, pp. 932-941, May 2012, doi: 10.1109/TPWRS.2011.2172821.

[16] P. Ojaghi and M. Rahmani, "LMI-Based Robust Predictive Load Frequency Control for Power Systems With Communication Delays," IEEE Trans. Power Syst., vol. 32, no. 5, pp. 4091-4100, Sep. 2017, doi: 10.1109/TPWRS.2017.2654453.

[17] M. Dehghani and S. K. Y. Nikravesh, "Decentralized nonlinear Ho controller for large scale power systems," Int. J. Electr. Power Energy Syst., vol. 33, no. 8, pp. 1389-1398, Oct. 2011, doi: 10.1016/j.ijepes.2011.06.019.

[18] K. Kiriakidis, "Robust stabilization of the Takagi-Sugeno fuzzy model via bilinear matrix inequalities," IEEE Trans. Fuzzy Syst., vol. 9, no. 2, pp. 269277, Apr. 2001, doi: 10.1109/91.919248.

[19] K. C. Goh, L. Turan, M. G. Safonov, G. P. Papavassilopoulos, and J. H. Ly, "Biaffine matrix inequality properties and computational methods," in Proceedings of 1994 American Control Conference - ACC '94, Jun. 1994, vol. 1, pp. 850-855 vol.1, doi: 10.1109/ACC.1994.751863.

[20] Q. T. Dinh, W. Michiels, S. Gros, and M. Diehl, “An inner convex approximation algorithm for BMI optimization and applications in control," in 2012 IEEE 51st IEEE Conference on Decision and Control (CDC), 2012, pp. 3576-3581.

[21] E. Kim and D. Kim, “Stability analysis and synthesis for an affine fuzzy system via LMI and ILMI: discrete case,” IEEE Trans. Syst. Man Cybern. Part B Cybern., vol. 31, no. 1, pp. 132-140, Feb. 2001, doi: 10.1109/3477.907572.

[22] F. Zheng, Q.-G. Wang, and T. H. Lee, “A heuristic approach to solving a class of bilinear matrix inequality problems,” Syst. Control Lett., vol. 47, no. 2, pp. 111-119, Oct. 2002.

[23] J.-C. Lo and M.-L. Lin, “Observer-Based Robust H/sub /spl infin//;Control for Fuzzy Systems Using Two-Step Procedure,” IEEE Trans. Fuzzy Syst., vol. 12, no. 3, pp. 350-359, Jun. 2004, doi: 10.1109/TFUZZ.2004.825992. 
[24] H. Javanmardi, M. Dehghani, M. Mohammadi, S. Siamak, and M. R. Hesamzadeh, "Bmi-based load frequency control in microgrids under false data injection attacks," IEEE Systems Journal, 2021.

[25] H. Javanmardi, M. Dehghani, M. Mohammadi, and N. Vafamand, "Bilinear matrix inequality-based nonquadratic controller design for polytopic-linear parameter varying systems," Int. J. Robust Nonlinear Control, vol. 30, no. 17, pp. 7655-7669, Nov. 2020, doi: 10.1002/rnc.5215.

[26] P. Apkarian and H. D. Tuan, "Low nonconvexity-rank bilinear matrix inequalities: algorithms and applications in robust controller and structure designs," IEEE Trans. Autom. Control, vol. 45, no. 11, pp. 2111-2117, Nov. 2000, doi: 10.1109/9.887636.

[27] Y.-J. Chen, K. Tanaka, M. Tanaka, S.-H. Tsai, and H. O. Wang, “A Novel Path-Following-Method-Based Polynomial Fuzzy Control Design,” IEEE Trans. Cybern., 2019.

[28] W.-Y. Chiu, "Method of Reduction of Variables for Bilinear Matrix Inequality Problems in System and Control Designs," IEEE Trans. Syst. Man Cybern. Syst., vol. 47, no. 7, pp. 1241-1256, Jul. 2017, doi: 10.1109/TSMC.2016.2571323.

[29] H. Saadat, Power Systems Analysis. McGraw-Hill, 2004.

[30] A. Kahrobaeian and Y. A.-R. I. Mohamed, "Direct Single-Loop/spl mu/-Synthesis Voltage Control for Suppression of Multiple Resonances in Microgrids with Power-Factor Correction Capacitors,” IEEE Trans. Smart Grid, vol. 4, no. 2, pp. 1151-1161, Jun. 2013, doi: 10.1109/TSG.2012.2228014.

[31] I. Zamani and M. H. Zarif, "On the continuous-time Takagi-Sugeno fuzzy systems stability analysis,” Appl. Soft Comput., vol. 11, no. 2, pp. 2102-2116, 2011.

[32] S. Falahati, S. A. Taher, and M. Shahidehpour, "Grid frequency control with electric vehicles by using of an optimized fuzzy controller," Appl. Energy, vol. 178, pp. 918-928, Sep. 2016, doi: 10.1016/j.apenergy.2016.06.077.

[33] M. R. Khalghani, M. H. Khooban, E. Mahboubi-Moghaddam, N. Vafamand, and M. Goodarzi, "A self-tuning load frequency control strategy for microgrids: Human brain emotional learning," Int. J. Electr. Power Energy Syst., vol. 75, pp. 311-319, Feb. 2016, doi: 10.1016/j.ijepes.2015.08.026.

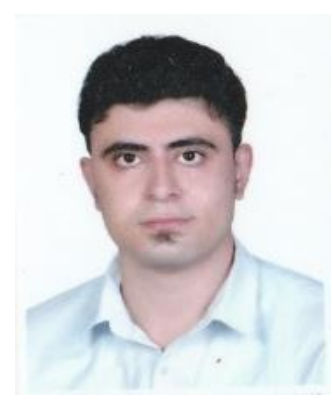

Hamidreza Javanmardi received his B. Sc. and M. Sc. degrees in Electrical Engineering from Shiraz University, Shiraz, Iran, in 2013 and 2015. He is currently a Ph.D. Student in Electrical Engineering at Shiraz University, Shiraz, Iran. He is interested in Model Predictive Control, Bilinear Matrix Inequality, automation and instruments, robotic and artificial intelligence.

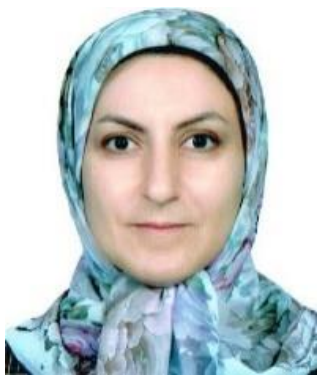

Maryam Dehghani (M'10-SM'18) received her B. Sc. and M. Sc. degrees in electrical engineering from Shiraz university, Shiraz, Iran, in 1999 and 2002, respectively and her PhD from Amirkabir University of Technology, Tehran, Iran in 2008. She is currently an associate professor in the school of electrical and computer engineering, Shiraz university, Shiraz, Iran. Her research interests include system modeling, estimation, control and their applications in power systems, energy systems and

biomedical systems. 


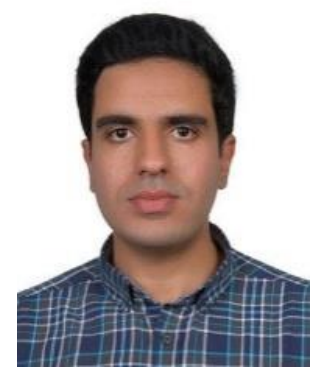

Mohsen Mohammadi received his Ph.D. degree in Mechanical Engineering from Shiraz University in 2018. He has been a faculty member with the school of mechanical engineering, Shiraz University, Shiraz, Iran, since then. His research interests include optimization, stochastic control, advanced mathematics, and mechatronics.

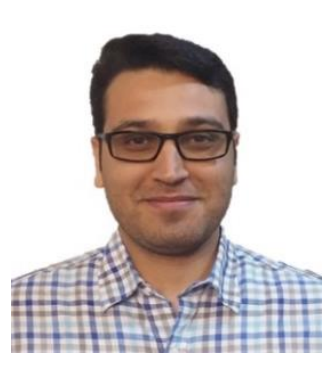

Navid Vafamand received his B.Sc. degree in electrical engineering and M.Sc. degree in control engineering form Shiraz University of Technology, Iran, in 2012 and 2014, respectively, and Ph. D. in control engineering at Shiraz University, Shiraz, Iran, in 2019. Currently, he serves as a research assistance at Shiraz University. He was a Ph.D. Visiting student with the Department of Energy Technology, Aalborg University, Denmark, from 2017 to 2018. Dr. Vafamand is the co-author of more than 80 international conference and journal papers and two chapter-books and an active reviewer in several journals. His main research interests include Takagi-Sugeno (TS) fuzzy systems, linear parameter varying (LPV) models, predictive control, and DC microgrids.

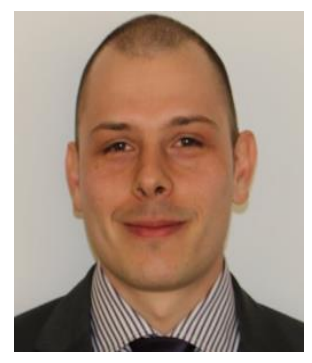

Tomislav Dragičević (S'09-M'13-SM'17) received the M.Sc. degree and the industrial Ph.D. degrees in electrical engineering from the Faculty of Electrical Engineering, University of Zagreb, Zagreb, Croatia, in 2009 and 2013, respectively.,From 2013 to 2016, he was a Postdoctoral Research Associate with Aalborg University, Denmark, where he was an Associate Professor from 2016 to 2020. He was a Guest Professor at Nottingham University, U.K., during Spring/Summer of 2018. Since 2020, he has been a Professor with the Technical University of Denmark. His research interests include design and control of microgrids, and 
application of advanced modeling and control concepts to power electronic systems. He has authored and coauthored more than 200 technical papers (more than 100 of them are published in international journals, mostly in IEEE), eight book chapters, and a book in the field.,Dr. Dragičević is an Associate Editor of the IEEE Transactions on Industrial Electronics, IEEE Transactions on Power Electronics, IEEE Journal of Emerging and Selected Topics in Power Electronics, and IEEE Industrial Electronics Magazine. He was a recipient of the Končar Prize for the Best Industrial Ph.D. thesis in Croatia, and a Robert Mayer Energy Conservation Award. He is a winner of Alexander van Humboldt fellowship for experienced researchers. 